\section{Infecção pelo HIV entre usuários de drogas injetáveis: análise dos fatores de risco no Município do Rio de Janeiro, Brasil}

\section{HIV-infection among injecting drug users: analysis of risk factors in Rio de Janeiro City, Brazil.}

\section{Paulo Roberto Telles}

Programa de Engenharia Biomédica COPPE-UFRJ e Núcleo de Estudos em Atenção ao Uso de drogas (NEPAD-UERJ)

Endereço para correspondência:

NEPAD/UERJ

R. Fonseca Teles, $121,4^{\circ}$ andar

20940-200 Rio de Janeiro, RJ - Brasil

\section{Renan Moritz R.Varnier-Almeida}

Programa de Engenharia Biomédica COPPE-UFRJ

Rio de Janeiro, RJ - Brasil

\section{Francisco Inácio Bastos}

Departamento de Informações para a Saúde da Fundação Oswaldo Cruz (DIS/CICT/FIOCRUZ)

Rio de Janeiro, RJ - Brasil

\section{Auxílio financeiro}

Ministério da Saúde/Coordenação Nacional de DST/AIDS e Programa de AIDS da Fundação Oswaldo Cruz (PIAFI/FIOCRUZ)

Testes laboratoriais realizados no Departamento de Imunologia, Fundação Oswaldo Cruz (IOC/FIOCRUZ), sob a responsabilidade da Dra. Mariza G. Morgado. Apoio da Fundação de Amparo à Pesquisa do Rio de Janeiro - FAPERJ (PRT) e do Conselho Nacional de Desenvolvimento Científico e Tecnológico CNPq (FIB).

\section{Resumo}

O trabalho visa a determinar os fatores de risco para a infecção pelo HIV (Human Immunodeficiency Virus - vírus da aids) entre usuários de drogas injetáveis (UDI), no Município do Rio de Janeiro. Os entrevistados foram recrutados em centros de tratamento para usuários de drogas e nas "cenas de uso" ("ruas"). Foram utilizados três questionários: um destinado a colher informações mais gerais acerca dos UDI, adaptado de estudo anterior da Organização Mundial da Saúde (OMS); um segundo voltado para a pesquisa de informações suplementares sobre conhecimentos, comportamentos e atitudes em relação à aids; além de um terceiro instrumento destinado a avaliar níveis de disfunção psicológica. O estudo consistiu de 110 entrevistados que voluntariamente realizaram testes laboratoriais para a presença do HIV, além de outros agentes infecciosos de transmissão sangüínea (HBV, HCV, HDV, HGV/GBVC, HTLV-I/II). A soroprevalência para o HIV-1 foi de $28,7 \%$. Utilizando-se um modelo de regressão logística múltipla (Estatística de Hosmer-Lemeshow, $\mathrm{c}^{2}=1,89$; $\mathrm{p}=0,98$ ), foram identificados como fatores de risco para a infecção pelo HIV: "local de moradia de baixa renda" (OR=5,57; IC 95\%: [1,39 - 22,27]), "fonte de renda irregular" (OR=3,26; IC 95\%: [1,01 - 10,51]), "menor idade de início quando do consumo de drogas injetáveis" (OR=2,50; IC: 90\%: [0,99 $6,28]$ ), "local de recrutamento nos centros de tratamento para uso de drogas" (vs. recrutamento nas "ruas") (OR=7,91; IC 95\%: [2,03 - 30,87]) e "maior freqüência de compartilhamento do material de injeção nos 6 meses anteriores à entrevista" $(\mathrm{OR}=4,41$; IC 95\%: [1,33 - 14,64]).

Palavras-chave: Infecções por HIV. Usuários de drogas injetáveis. Fatores de risco. Abuso de substâncias por via endovenosa. Síndrome de Imunodeficiência Adquirida. 


\section{Abstract}

The objective of the present paper is to identify risk factors for HIV infection among injecting drug users in the City of Rio de Janeiro. A survey was carried out with volunteers selected from (drug use) treatment centers and from street based drug users of the city. Three questionnaires were used: the first one addressed information on general aspects of the population of injecting drug users; the second one asked for additional information on HIV-related knowledge and behavior; and a third one was designed to assess psychological dysfunction. The sample consisted of 110 respondents, who volunteered for HIV-infection and other blood-borne pathogens (HBV, HCV, HDV, HGV/GBV-C, HTLV-I/II) laboratory testing. In the sample, HIV-1 point seroprevalence was $28.7 \%$. A logistic regression model (Hosmer-Lemeshow statistics, $\left.\mathrm{c}^{2}=1.89 ; \mathrm{p}=0.98\right)$ identified the following variables as risk factors for HIV infection: "low-income residence" (OR=5.57; 95\% CI: [1.39 - 22.27]), "uncertain income sources" (OR=3.26; 95\% CI: [1.01 - 10.51]), "early onset of drug consumption" (OR=2.50; 90\% CI: [0.99 - 6.28]), "recruitment from street based drug users" (OR=7.91; 95\% CI: [2.03 30.87]), and "high frequency of needle-sharing during the prior 6 months" ( $\mathrm{OR}=4.41$; 95\% CI: [1.33 - 14.64]).

Keywords: HIV-infections. Risk factors. Substance abuse, intravenous. Acquired Immunodeficiency Syndrome.

\section{Introdução}

O Brasil é um dos países com o maior número de casos notificados de aids no mundo. Dos mais de 120.000 casos de aids registrados até maio de 1998, pouco menos de um quarto ocorreu entre usuários de drogas injetáveis (UDI) ${ }^{1}$. Os UDI também têm um importante papel na transmissão sexual e vertical do HIV, observando-se que $23 \%$ dos casos de aids entre mulheres nos cinco últimos anos têm o uso de drogas injetáveis como categoria de exposição e que, dentre as infecções secundárias à transmissão heterossexual, 33\% delas tiveram um UDI como parceiro sexual ${ }^{2,3}$. Além da transmissão do HIV, as práticas de injeção de drogas facilitam a difusão de outras doenças infecciosas e problemas clínicos, como as hepatites virais $(B, C$, a recém identificada hepatite $\mathrm{G}$, além da hepatite delta, presente em UDI americanos e europeus $^{4}$, mas ainda não identificada no país); as infecções pelo HTLV-I/II e a tuberculo$\mathrm{se}^{5,6}$.

O Rio de Janeiro é o segundo estado brasileiro em número de casos notificados de aids $^{1}$. Estudos realizados especificamente com UDI na cidade do Rio de Janeiro ${ }^{7-10}$ mostraram taxas de soroprevalência para o HIV em torno de $30 \%$, com pequenas variações, exceção feita à amostra do Projeto coordenado pelo NEPAD-UERJ/Universidade de Miami que, recrutando apenas amostras oriundas das "cenas de uso" ("ruas"), em áreas de baixa renda distribuídas por todo o município do Rio de Janeiro, encontrou uma prevalência significativamente menor $-14 \%^{11}$. Também foram encontrados em todos os estudos elevados índices de comportamentos de risco, tais como o compartilhamento de seringas e o sexo não protegido ${ }^{9,10}$.

A fundamentação de estratégias de prevenção para a infecção pelo HIV, assim como sua adequada avaliação, seja entre UDI, ou em outras populações, se baseia, de um modo geral, em dados empíricos obtidos por meio da análise de inquéritos populacionais ("surveys") realizados nas 
populações-alvo. Devido ao fato dos principais fatores de risco conhecidos serem de ordem comportamental, tais levantamentos constam de extensos questionários, cujas questões (variáveis) aferem múltiplos aspectos (conhecimentos, práticas, atitudes e crenças) das populações estudadas.

O presente trabalho tem como objetivo analisar os padrões comportamentais e elementos que compõem o perfil psicológico de uma população de UDI, procurando identificar aqueles fatores associados a um aumento no risco de infecção pelo HIV, a partir de dados levantados no segmento regional (desenvolvido no Rio de Janeiro) de um Estudo Multicêntrico (Projeto Brasil), apoiado e parcialmente financiado pelo Ministério da Saúde. Os resultados obtidos visam a auxiliar a seleção das estratégias de prevenção entre UDI, assim como estimar o impacto daquelas já implementadas, como os programas de troca de seringas e agulhas.

\section{Metodologia}

\section{População}

Foram analisados dados obtidos a partir da aplicação de três questionários a uma amostra de 110 UDI. Os critérios de inclusão no estudo foram o uso de drogas injetáveis nos 6 meses que antecederam a data da entrevista e ter mais de 16 anos de idade, sendo, portanto o(a) entrevistado(a) capaz de assinar termo de consentimento informado, prévio à inclusão do (a) entrevistado(a) no estudo. O termo de consentimento informado foi desenvolvido segundo as normas éticas adotadas no Brasil e EUA para pesquisas realizadas em seres humanos, ressaltando ser esta uma pesquisa voluntária, podendo o participante desistir da mesma em quaisquer de suas etapas.

O recrutamento foi realizado no período de 1994 a 1996, como parte de um Projeto Multicêntrico, encerrado em dezembro de 1996. Foi utilizada uma estratégia mista de recrutamento, incluindo usuários de drogas provenientes das "ruas" (nãoinstitucionalizados), e de centros de trata- mento para o uso de drogas. Os usuários das "ruas" foram recrutados de diversas fontes, incluindo locais públicos, clubes noturnos e bares, distribuídos por várias regiões do Município do Rio de Janeiro. Recrutadores foram especialmente treinados para esta tarefa, sendo o preenchimento do questionário realizado de modo a preservar a privacidade dos participantes. Os UDI provenientes de centros de tratamento foram recrutados basicamente de três unidades de tratamento para usuários de drogas: o "Centro de Recuperação de Dependência Química (CREDEQ)" (localizado em Campo Grande, Zona Oeste do Rio de Janeiro), a "Unidade Certa" (Seção Paracambi, município vizinho, mas com clientela basicamente proveniente do Município do Rio de Janeiro) e o "Núcleo de Estudos e Pesquisas em Atenção ao Uso de Drogas da Universidade do Estado do Rio de Janeiro (NEPAD/UERJ)" (São Cristovão, Zona Norte do Rio de Janeiro). Ofereceu-se uma pequena ajuda financeira aos participantes $(\mathrm{R} \$ 6,00)$ para cobrir despesas de transporte e um lanche. Foi colhida uma amostra de sangue, de $20 \mathrm{ml}$, para posterior testagem sorológica para o HIV e demais patógenos de transmissão sangüínea. Exceção feita a dois entrevistados, todos os demais concordaram em ser testados.

\section{Questionários}

O primeiro questionário, básico, abrangia dados sócio-demográficos, padrões de consumo de drogas, hábitos sexuais, conhecimentos acerca da aids e resultados de teste(s) anterior(es) para o HIV. Aplicado em todas as cidades que integraram o Estudo Multicêntrico Brasileiro, este questionário foi desenvolvido pela equipe do Projeto Brasil, a partir de experiência anterior no âmbito do Estudo Multicêntrico da OMS (Multi-City Study on Drug Injecting and Risk of HIV infection) ${ }^{9}$.

O segundo, desenvolvido por um dos Autores do presente trabalho (PRT), em cooperação com o "Center for AIDS Prevention Studies”, de São Francisco, vi- 
sava obter informações complementares ao primeiro questionário, sobre os hábitos de vida dos entrevistados, especialmente aqueles vinculados ao consumo de drogas (detalhes acerca dos padrões de uso injetável, motivações para o compartilhamento do equipamento de injeção, forma como cada UDI avalia sua vulnerabilidade); hábitos sexuais (parceiros sexuais, comportamentos de risco com parceiros sexuais, motivações com relação ao uso de preservativos); além de outros conhecimentos, atitudes e comportamentos frente ao risco de infecção pelo HIV.

O terceiro e último questionário utilizado foi o GHQ-12 - General Health Questionnaire, versão com 12 perguntas ${ }^{12}$. Trata-se de um questionário de rápida aplicação e já validado em nosso meio ${ }^{13}$. O GHQ-12 tem como propósito avaliar o grau de disfunção psicológica (sem, contudo, caracterizar a natureza desta disfunção) em entrevistados inseridos em contextos comunitários ou em serviços de atenção primária e outras unidades de saúde não especializadas (não-psiquiátricas) ${ }^{12}$. A partir de cada questionário preenchido obtémse uma pontuação que pode variar de $0 \mathrm{a}$ 12. Maiores pontuações sugerem maiores chances de estarem presentes distúrbios psicológicos no entrevistado. Também é possível o emprego de diferentes pontos de corte (limiares) nestas pontuações, para que os entrevistados possam ser separados em dois grupos, um com maior e outro com menor chance de possuir distúrbios psicológicos. Neste trabalho foram definidos $5 /$ 6 pontos como ponto de corte, agrupando os entrevistados com pontuações maiores que cinco (supostamente, portadores de disfunção psicológica), e comparados àqueles com pontuações menores ou iguais a cinco. O método de pontuação e determinação de limiares segue metodologia sugerida pelos autores do questionário ${ }^{12}$.

\section{Testes laboratoriais}

As amostras de sangue foram testadas para a presença de anticorpos para o HIV pelo laboratório de Imunologia do IOC, FIOCRUZ. Seguiu-se o algoritmo de teste definido pelo Ministério da Saúde ${ }^{14}$, com a realização de testes Elisa (Abbott HIV-1 recombinant EIA, $3^{\text {rd }}$ gen., Abbott Laboratories, Diagnostic Division North Chicago, USA) e dois testes confirmatórios, de Imunofluorescência (Biomanguinhos, Fundação Oswaldo Cruz, Rio de Janeiro, Brasil) e Western Blot (Cambridge Biotech. Corp., Worcester, MA, USA).

\section{Plano de análise dos dados}

Inicialmente, estabeleceu-se a associação individual (análise univariada, teste $\chi^{2}$ ) entre a sorologia para o HIV e as demais variáveis demográficas e comportamentais coletadas. Todas as variáveis foram dicotomizadas antes da aplicação do teste. A seguir, foi desenvolvido um modelo de regressão logística para explorar os preditores da sorologia para o HIV, utilizando a sorologia (positiva/negativa) como variável dependente. A seleção das variáveis independentes a serem preliminarmente incluídas no modelo se baseou em dois critérios: a) variáveis que apresentaram associações estatisticamente significantes (nível de significância considerado de 0,10 ) na análise univariada (Tabela 2); e b) variáveis tradicionalmente associadas à sorologia positiva para o HIV, ou seja, que apresentaram associações estatisticamente significantes em estudos semelhantes ${ }^{7,10,15}$. Tais variáveis em geral representavam comportamentos de risco diretamente ligados à infecção, como compartilhamento do equipamento de injeção, hábitos sexuais de risco, idade do início do uso e tempo de uso de drogas injetáveis.

As variáveis foram mantidas no modelo a partir do julgamento de sua importância e adequação como preditores da variável dependente e seu nível de significância estatística no modelo final. Foi utilizado como nível de significância estatística para manutenção das variáveis no modelo, $\mathrm{p}=0,05$; exceção feita a uma variável - "início do consumo de drogas injetáveis antes dos 18 
anos de idade", onde se utilizou $\mathrm{p}=0,10$ (ver discussão). Como as variáveis "local de moradia de baixa renda" e "não ter fonte de renda regular" referem-se a conceitos semelhantes (ambos apontando para o nível sócio-econômico do entrevistado), e apresentaram associação estatisticamente significante, foi testada no modelo logístico a inclusão de um termo de interação entre eles, o qual não apresentou associação com a variável dependente $(\mathrm{p}=0,95)$, não sendo, portanto, incluído no modelo final. A estatística de Hosmer-Lemeshow foi utilizada para análise de adequação do modelo logístico final ${ }^{16}$. O programa estatístico utilizado foi o SPSS (Statistical Package for the Social Sciences), versão 7.5 ${ }^{17}$.

\section{Resultados}

A Tabela 1 apresenta as características sociodemográficas e comportamentais (uso de drogas e hábitos sexuais) observadas na amostra. Deve ser assinalado o elevado índice de comportamentos de risco encontrado. As amostras de sangue para os testes sorológicos foram obtidas de 108 entrevistados, com uma soroprevalência para o HIV de $28,7 \%$.

Na Tabela 2, apresentam-se as principais associações individuais entre a sorologia para o HIV e as variáveis sociodemográficas e comportamentais. As variáveis consideradas como apresentando associação estatisticamente significante (valores-p em negrito na tabela) foram: "local de recrutamento"; "situação conjugal", "residência em local de baixa renda", "fonte de renda regular/autônomo", "compartilhamento de seringas e agulhas nos últimos 5 anos" e "tem parceiro sexual principal".

A Tabela 3 apresenta o modelo de regressão logística obtido, os Odds ratios ajustados e intervalos de confiança, além dos Odds ratios da análise univariada para cada uma das variáveis independentes. $\mathrm{O}$ teste de Hosmer-Lemeshow para a adequação do modelo forneceu $\chi^{2}=1,89 ; \mathrm{p}=0,98$ (Ho: o modelo se ajusta perfeitamente aos dados), implicando a aceitação do modelo.

\section{Discussão}

Algumas características socioeconômicas e comportamentais encontradas (Tabela 1), como, por exemplo, a distribuição por sexo, estrato social, nível de escolaridade, hábitos e comportamentos de risco, são comparáveis às encontradas em outros estudos realizados no Brasil ${ }^{6,7,9}$, e em pesquisas realizadas em outros países ${ }^{8,9,18}$.

A população estudada referiu altos níveis de comportamentos de risco. Por exemplo, o compartilhamento de seringas e o sexo sem preservativos foram freqüentemente citados, apesar de os UDI revelarem um bom conhecimento acerca dos mecanismos de transmissão da AIDS 93,3\% tinham conhecimento da possibilidade de transmissão do HIV por intermédio do compartilhamento de seringas e agulhas e, $98,1 \%$, através das relações sexuais.

Conhecer a dinâmica de transmissão do $\mathrm{HIV}$, incluindo seus fatores de risco mais relevantes, mesmo que em populações selecionadas, constitui mais uma peça na elaboração e implementação de medidas preventivas. Certas limitações de estudos desta natureza, são muito dificilmente contornáveis, pois envolvem o consumo de substâncias ilegais e têm como alvo uma população extremamente marginalizada, discriminada até mesmo por outros usuários de drogas (não injetáveis). Isto importa em uma grande dificuldade de localização, recrutamento e envolvimento dos participantes na pesquisa. Tais dificuldades são citadas na ampla maioria dos estudos com UDI ${ }^{19}$.

A despeito da elevada freqüência observada de práticas sexuais de risco, elas não se mostraram relevantes como fatores de risco no modelo final. Isto possivelmente se deveria à maior eficiência da transmissão do HIV pela via sangüínea, "encobrindo" os riscos adicionais de natureza sexual ${ }^{20}$, ou à relativa limitação dos instrumentos utilizados em captar a real dimensão dos riscos secundários às práticas sexuais, algo freqüentemente mencionado pela literatura relativa à população dos $\mathrm{UDI}^{21}$. 
Tabela 1 - Características sociodemográficas e comportamentais da população estudada (número de entrevistados = 110).

Table 1 - Socio-demographic and behavioral characteristics of the studied population (110 interviewees).

$\mathrm{N}(\%) *$

110

$110(15,5)$

109

$(50,5)$

$(49,5)$

$108(33,3)$

110

$(23,6)$

$(76,4)$

109

$(26,6)$

$(50,5)$

$(22,9)$

110

$(44,5)$

$(47,3)$

$(8,2)$

105

$(44,8)$

$(55,2)$

Classe média
Média (desvio padrão)

$33,7(7,9)$

Comportamentos quanto ao uso de drogas injetáveis

Idade de início do uso de drogas injetáveis (em anos):

Anos de uso de drogas injetáveis

105

105

$19,3(5,1)$

$105(78,6)$

Injeta há mais de 5 anos:

Injeta há mais de 10 anos:

$105(50,5)$

105

$(25,7)$

$(32,4)$

1 a 3 vezes por semana $(M)$

$110(56,5)$

Compartilhou agulhas/seringas nos últimos 6 meses:

$110(73,8)$

Comportamento sexual

Qualquer relacionamento sexual heterossexual nos últimos 30 dias:

№ de parceiros sexuais nos últimos 30 dias**:

$110(55,5)$

106

$14,1(9,0)$

Sempre usou preservativos nos últimos 30 dias:

$106(10,9)$

$103(51,8)$

Sexo por dinheiro ou drogas:

$91(50,9)$

$56(21,8)$

Qualquer relacionamento sexual homossexual nos últimos 6 meses:

$56(28,6)$

Sempre usou preservativos nas relações homossexuais***:

Questões acerca da AIDS

Envolvimento em atividades de prevenção:

Efetuou mudanças de comportamento após o surgimento da AIDS:

$105(41,9)$

$105(55,5)$

$104(93,3)$

Sabia da possível transmissão do HIV através de drogas injetáveis

$104(98,1)$

\footnotetext{
${ }^{*} \mathrm{~N}$ : número de respostas disponíveis; (\%): proporção de respostas positivas [N: number of available responses; (\%): proportion of positive answers. ** Relativo aos sexualmente ativos [of those sexually active]. ${ }^{* * *}$ Entre os que tiveram relacionamento homossexual nos 6 meses anteriores à entrevista. [among those who reported homosexual intercourse during the 6 months before the interview]
} 
Tabela 2 - Relacões univariadas entre a sorologia para o HIV e principais variáveis independentes, teste $\chi^{2}$

Table 2 - Univariate analysis of HIV serostatus and main independent variables, $\chi^{2}$ test.

\begin{tabular}{|c|c|c|c|}
\hline Variável & Categorias & $\mathrm{N}(\% \mathrm{HIV}+)$ & Valor de $\mathrm{p}$ \\
\hline \multirow[t]{2}{*}{ Idade: } & $\geq 30$ anos & $76(28,1)$ & 0,93 \\
\hline & $<30$ anos & $32(28,9)$ & \\
\hline \multirow[t]{2}{*}{ Sexo: } & Masculino & $92(29,3)$ & 0,72 \\
\hline & Feminino & $16(25,0)$ & \\
\hline \multirow[t]{2}{*}{ Nível de escolaridade: } & $<2^{\circ}$ grau completo & $53(24,5)$ & 0,42 \\
\hline & $\geq 2^{\circ}$ grau completo & $54(31,5)$ & \\
\hline \multirow[t]{2}{*}{ Local de recrutamento: } & Ruas & $26(42,3)$ & 0,08 \\
\hline & Eentro de Tratamento & $82(24,4)$ & \\
\hline \multirow[t]{2}{*}{ História de prisão: } & Sim & $79(29,1)$ & 0,68 \\
\hline & Não & $28(25,0)$ & \\
\hline \multirow[t]{2}{*}{ Situação conjugal: } & Vive só & $71(33,8)$ & 0,07 \\
\hline & ive c/ parceiro sexual & $35(17,1)$ & \\
\hline \multirow[t]{2}{*}{ Escore no "GHQ": } & $>5$ pontos & $66(28,8)$ & 0,81 \\
\hline & $\leq 5$ pontos & $34(26,5)$ & \\
\hline \multirow[t]{2}{*}{ Reside local de baixa renda: } & Sim & $69(36,2)$ & 0,02 \\
\hline & Não & $34(14,7)$ & \\
\hline \multirow[t]{2}{*}{ Fonte de renda regular/autônomo: } & Não & $51(41,2)$ & 0,007 \\
\hline & Sim & $48(16,7)$ & \\
\hline \multirow[t]{2}{*}{ Idade de início do uso: } & $<18$ & $44(36,4)$ & 0,11 \\
\hline & $\geq 18$ & $59(22,0)$ & \\
\hline \multirow[t]{2}{*}{ Tempo de uso injetável: } & $>10$ anos & $52(26,9)$ & 0,78 \\
\hline & $\leq 10$ anos & $51(29,4)$ & \\
\hline \multirow[t]{3}{*}{ Freqüência de injeção: } & Baixa & $27(33,3)$ & 0,25 \\
\hline & Moderada & $34(17,6)$ & \\
\hline & Alta & $42(33,3)$ & \\
\hline \multirow[t]{2}{*}{ Compartilhou nos últimos 5 anos: } & Sim & $76(35,5)$ & 0,005 \\
\hline & Não & $27(7,4)$ & \\
\hline \multirow[t]{2}{*}{ Compartilhou nos últimos 6 meses: } & Sim & $61(34,4)$ & 0,13 \\
\hline & Não & $47(21,3)$ & \\
\hline \multirow{2}{*}{$\begin{array}{l}N^{\circ} \text { de parc. heterossexuais } \\
\text { (último mês) }\end{array}$} & $>3$ & $54(31,5)$ & 0,40 \\
\hline & $\leq 3$ & $50(24,0)$ & \\
\hline \multirow[t]{2}{*}{ Tem parceiro principal: } & Sim & $60(20,0)$ & 0,04 \\
\hline & Não & $44(38,6)$ & \\
\hline \multirow[t]{2}{*}{ Tem parceiro ocasional: } & Sim & $66(28,8)$ & 0,78 \\
\hline & Não & $38(26,3)$ & \\
\hline \multirow[t]{2}{*}{ Tem clientes: } & Sim & $56(26,8)$ & 0,81 \\
\hline & Não & $45(28,9)$ & \\
\hline \multirow{2}{*}{$\begin{array}{l}\text { Relações homossexuais: } \\
\text { (últimos } 5 \text { anos, sexo masculino) }\end{array}$} & Sim & $56(26,8)$ & 0,57 \\
\hline & Não & $34(32,4)$ & \\
\hline \multirow{2}{*}{$\begin{array}{l}\text { Mudou de comportamento: } \\
\text { (p/ evitar a infecção pelo HIV) }\end{array}$} & Não & $44(31,8)$ & 0,36 \\
\hline & Sim & $59(23,7)$ & \\
\hline
\end{tabular}


Tabela 3 - Preditores para sorologia positiva para o HIV no modelo de regressão logística, Odds ratios brutos e ajustados e Intervalos de Confiança.

Table 3 - HIV+ predictors in the logistic model, unadjusted and adjusted Odds ratios, and confidence intervals

\begin{tabular}{lccc}
\hline & $\begin{array}{c}\text { Odds ratio } \\
\text { bruto (análise } \\
\text { univariada) }\end{array}$ & $\begin{array}{c}\text { Odds ratio } \\
\text { modelo } \\
\text { multivariado }\end{array}$ & $\begin{array}{c}\text { IC 95\% } \\
\text { (modelo } \\
\text { multivariado) }\end{array}$ \\
\hline Residir em áreas de baixa renda & 3,29 & 5,57 & $1,39-22,27$ \\
Não ter fonte de renda regular & 3,50 & 3,26 & $1,01-10,51$ \\
Início do consumo (drogas injetáveis) < 18 anos & 2,02 & 2,50 & $0,99-6,28^{*}$ \\
Ter sido recrutado nas "ruas" & 2,27 & 7,91 & $1,39-22,27$ \\
Maior compartilhamento (6 meses anteriores) & 1,91 & 4,41 & $1,33-14,64$ \\
\hline
\end{tabular}

$*$ IC $90 \%$

A diferença estatisticamente significante das taxas de infecção pelo HIV, encontradas segundo os diferentes locais de recrutamento ("ruas" vs. centros de tratamento), também foi encontrada em outros estudos $^{7,9}$, indicando a importância de um recrutamento específico para a população das "ruas", de acesso mais difícil e com características comportamentais menos conhecidas. Este fato talvez constitua uma das explicações para uma soroprevalência para o HIV significativamente mais elevada encontrada na cidade de Santos, em uma amostra composta exclusivamente por entrevistados recrutados nas "ruas" ${ }^{6}$. Paradoxalmente, uma amostra de UDI de Projeto coordenado pelo NEPAD-UERJ/Universidade de Miami, no Município do Rio de Janeiro, que recrutou apenas amostras oriundas das "ruas", pertencentes aos estratos sociais mais pobres (residentes em favelas), encontrou uma soroprevalência para o HIV significativamente menor $-14 \%{ }^{11}$. Ainda são desconhecidas as razões dessa discrepância, podendo ser decorrentes de variações da soroprevalência para o HIV de acordo com a região de moradia no Estado, aliada à possibilidade de existirem diferentes velocidades de propagação do HIV nos diversos estratos socioeconômicos ${ }^{2}$.

Conforme esperado, dada a natureza dos riscos intrínsecos a que está sujeito este segmento e a eficácia da transmissão sangüínea do HIV, uma "freqüência maior de compartilhamento" de apetrechos de injeção nos 6 meses anteriores à entrevista foi um dos preditores de sorologia positiva para o HIV (Tabela 3).

Entre os UDI estudados, aqueles que se iniciaram mais precocemente no uso de drogas injetáveis apresentaram maior probabilidade de se infectarem pelo HIV (variável “início do consumo...”). Optou-se por manter esta variável no modelo, mesmo tendo ela apresentado significância estatística marginal (nível de significância de 0,10), uma vez que em trabalhos semelhantes ${ }^{22}$, realizados em amostras maiores de UDI, este foi considerado um comportamento de risco associado de forma significativa à infecção pelo HIV.

Uma possível explicação para o impacto desta variável seria a menor quantidade de informações sobre prevenção e a menor maturidade entre os mais jovens e, conseqüentemente, um maior risco de infecção pelo HIV nesta fase ${ }^{23}$. Cabe ressaltar que a idade média de início de uso para o grupo de indivíduos infectados pelo HIV foi de 18,1 anos, e que usuários de drogas mais jovens, em outros estudos, haviam referido mais freqüentemente o hábito do compartilhamento de agulhas e seringas (10).

A taxa de infecção pelo HIV foi mais baixa entre os usuários que dispunham de uma fonte de renda regular (emprego fixo ou autônomo), e entre aqueles que residiam em áreas de renda mais elevada. Explicações para este resultado emergem tanto da tendência epidemiológica mais geral, descrita 
em outros trabalhos ${ }^{24,25}$ - o "empobrecimento" da epidemia de AIDS no Brasil -, como do mesmo fenômeno, visto sob uma perspectiva micro-social - a maior vulnerabilidade de indivíduos e grupos pouco assistidos, providos de menores recursos (inclusive para adquirir preservativos e seringas estéreis) e alvo de estigma e discriminação. A epidemia se iniciou no País nas classes média e média-alta, movendo-se gradualmente para classes com ocupações menos especializadas, com menor nível de instrução, vivendo em regiões de renda mais baixa das grandes cidades brasileiras (e, mais recentemente, nas cidades de médio e pequeno porte). Diversos trabalhos, inclusive aqueles especificamente desenvolvidos entre UDI, apontam para uma maior vulnerabilidade de indivíduos e grupos com inserção social mais precária ${ }^{26,27}$.

Um outro achado importante refere-se aos escores encontrados no questionário que avaliou o estado psicopatológico dos entrevistados. Não foram encontradas diferenças estatisticamente significantes nos escores do GHQ entre os infectados e não infectados. Com isso, o emprego do GHQ12 não se mostrou eficaz como estratégia de "screening" entre os UDI infectados e não-infectados neste segmento populacional. Porém, o que chama, de fato, a atenção, são os escores elevados encontrados de uma maneira geral, muito superiores aos encontrados em outras populações ${ }^{12,13}$, indicando elevados níveis de disfunção psicológica entre os UDI, com repercussões, uma vez mais, sobre as estratégias de prevenção.

Como mostram outros estudos ${ }^{28}$, intervenções preventivas entre UDI podem ser eficazes na redução de comportamentos de risco, especialmente aquelas relativas aos hábitos de injeção. Infelizmente, o Rio de Janeiro só muito recentemente passou a dispor de um programa de prevenção sistemático para a AIDS entre UDI. O Ministério da Saúde vem implementando um programa de redução de danos no Município do Rio de Janeiro, junto com outras cidades brasileiras ${ }^{29}$. Esses programas têm encontrado uma substancial oposição política. Os achados neste estudo enfatizam que a implementação plena de tais programas é urgente, de modo a prevenir a disseminação do HIV entre os usuários de drogas e o restante da população. Cabe ressaltar que, apesar da importância do hábito do uso de drogas injetáveis com relação à dinâmica da epidemia pelo HIV/AIDS em nosso País, ainda são poucos os trabalhos sobre este tema em nosso meio, sendo necessários estudos mais aprofundados para que, a partir deles, sejam desenhadas estratégias de prevenção mais eficazes.

\section{Summary}

Injecting drug users (IDUs) play a very significant role in the dynamics of Brazilian HIV/AIDS epidemic, and this exposure category was responsible for almost $25 \%$ of the Brazilian AIDS cases registered as of May 1998. IDUs also play a central role in the further sexual spread of HIV to the general population. IDUs also comprise a population specially affected by other blood borne infections.

The objective of the present paper is to identify risk factors for HIV infection among injecting drug users in the City of Rio de Janeiro. A survey was carried out with volunteers selected from (drug use) treatment centers and from the "street based drug users" of the city. Three questionnaires were used: the first one addressed information on general aspects of the population of injecting drug users; the second one asked for additional information on HIV-related knowledge and behavior; and a third one called GHQ-12, a questionnaire specifically designed to assess psychological dysfunction 
in non-psychiatric settings. The sample consisted of 110 respondents, who volunteered for HIV-infection and other blood-borne pathogens ( $H B V, H C V, H D V, H G V / G B V-C$, $H T L V-I / I I)$ laboratory testing. In the sample, $H I V-1$ point seroprevalence was $28.7 \%$.

Contingency tables were built, assessing univariate associations between socio-demographic variables and HIV serostatus. The selection of independent variables to be included in the logistic multivariate analyses followed two complementary criteria: variables that had shown to be statistically associated with HIV infection in the univariate analysis (considering $p<0.10$ ), as well as variables formerly described in the international literature to be associated with HIV infection in this population.

A logistic regression model (HosmerLemeshow statistics, $c^{2}=1.89 ; p=0.98$ ) identified the following variables as risk factors for HIV infection: "low-income residence" (OR=5.57; 95\% CI: [1.39 - 22.27]), "uncertain income sources" (OR=3.26; 95\% CI: [1.01 10.51]), "early onset of drug consumption"
(OR=2.50; 90\% CI: [0.99 - 6.28]), "recruitment from street based drug users" (streets) (OR=7.91; 95\% CI: [2.03 - 30.87]), and "high frequency of needle-sharing during the prior 6 months" (OR=4.41; 95\% CI: [1.33 14.64]).

Despite the fact that the substantial majority of the sample had shown a sound knowledge on HIV transmission routes and ways to prevent HIV infection, most of them still engaged in high risk behavior. IDUs here analyzed presented high levels of sexual risk behavior, although none of these variables remained in the final logistic model. This seems to be secondary to the also high levels of risk behaviors associated with injection habits, and the greater efficiency of parenteral route in the transmission of HIV.

Preventive measures are needed urgently, addressing sexual risks, injecting risk behaviors, transmission of HIV and other blood borne infections in this population, their non-injecting sexual partners and offspring.

\section{Referências}

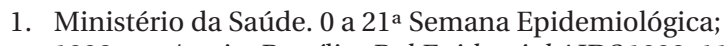
1998 mar/maio. Brasília. Bol Epidemiol AIDS 1998; 11(2).

2. Kalichman A. AIDS and intravenous drug use in Brasil. In: Monteiro MG, Inciardi JA, editors. Brasil - United States binational research. São Paulo: CEBRID/NIDA 1993. p. $49-62$.

3. Castilho E, Gomes M, Chequer P, Rodrigues L et al. Fifteen years of AIDS in Brazil: Epidemiological features and trends of adult females AIDS cases. In: Abstracts of the XI International Conference on AIDS; 1996 Jul; Vancouver. Abstract Mo.C. 450.

4. Kreek MJ, Des Jarlais DC, Trepo CL et al. Contrasting prevalence of Delta hepatitis markers in parenteral drug abusers with and without AIDS. J Infect Dis 1990; 162:538-41.

5. Kritsky A, Dalcolmo M, Del Bianco R et al. Associação tuberculose e infecção pelo HIV no Brasil. Bol Oficina Sanit Panam 1995; 118:542-54.

6. Carvalho HB, Mesquita F, Massad E et al. HIV and infections of similar transmission patterns in a drug injectors community of Santos, Brazil. J AIDS 1996; 12:84-92.

7. Lima ES, Friedman SR, Bastos FI, Telles PR et al. Risk factors for HIV-1 seroprevalence among drug injectors in the cocaine-using environment of Rio de Janeiro. Addiction 1994; 89:689-98.

8. Des Jarlais DC, Friedman SR, Friedmann P et al. HIV/ AIDS-related behavior change among injecting drug users in different national settings. AIDS 1995; 9:611-7.

9. WHO International Collaborative Group. Multi-City study on drug injecting and risk of HIV infection. Geneva: WHO; 1994.

10. Telles PR, Bastos FI, Guydish J et al. Risk behavior and HIV seroprevalence among IDUs in Rio de Janeiro, Brazil. AIDS 1997; 11 Suppl. 1: s35-s42.

11. Inciardi J, Surrat H, Telles P et al. Risks for HIV-1 infection and seropositivity rates among cocaine users in Rio de Janeiro, Brazil. In: Abstracts of the XI International Conference on AIDS; 1996; Vancouver. Abstract Th.C.425. 
12. Goldberg D, Williams, P. A user's guide to the general health questionnaire. London: Nfer-Nelson; 1988.

13. Mari JJ, Williams P. A comparison of the validity of two psychiatric screening questionnaires in Brazil using ROC analysis. Psychol Med 1985; 15: 651-9.

14. Ministério da Saúde. $36^{\text {a a }} 48^{\text {a }}$ Semana Epidemiológica; 1995 set/nov; Brasília. Bol Epidemiol AIDS 1995; 8(4).

15. Hartgers C, Van den Hoek JAR, Coutinho RA, Van der Pligt J. Psychopathology, stress and HIV-risk injecting behavior among drug users. Br J Addiction 1992; 87:85765 .

16. Hosmer DW, Lemeshow S. Applied logistic regression. New York: John Wiley; 1989.

17. Norusis MJ. SPSS 7.5 Guide to data analysis. Upper Saddle River (NJ): Prentice Hall; 1997.

18. Davoli M, Perucci CA, Abeni DD et al. HIV risk-related behaviors among injecting drug users in Rome: Differences between 1990 and 1992. Am J Public Health 1995; 85:829-32.

19. Frischer M, Golberg D, Green S. How many drug injectors are there in the UK. Int J Drug Policy 1993; 4:190-3.

20. Coppola RC, Masia G, Di Martino ML et al. Sexual behaviour and multiple infection in drug abusers. Eur J Epidemiol 1996; 12:429-35.

21. Donoghoe MC. Sex, HIV and the injecting drug user. $\mathrm{Br} J$ Addiction 1992; 87:405-16

22. Hartgers C, Van den Hoek JAR, Krijnen P et al. HIV prevalence and risk behavior among injecting drug users who participate in "low-threshold" methadone programs in Amsterdam. Am J Public Health 1992; 82:459.

23. Stark K, Müller R, Guggenmöos-Holzman I et al. HIV infection in intravenous drug abusers in Berlin: Risk factors and time trends. Klin Wochenschr 1990; 68:41569.

24. Bastos FI, Telles PR, Castilho EA, Barcellos C. A epidemia de AIDS no Brasil. In: Minayo MCS, organizador. Os muitos Brasis: saúde e população na década de 80. São Paulo: Hucitec/Rio de Janeiro: ABRASCO; 1995. p. 24568.

25. Bastos FI, Stimson G, Telles PR et al. Cities responding to the epidemics. In: Stimson G, Des Jarlais DC, Ball A, editors. Drug injecting and HIV infection: global dimensions and local responses. London: UCL Press; 1998. p.149-67.

26. Dobler-Michola A, Höfler DZ, Korbel R, Uchtenhagen A. HIV-risk and social integration in groups of drug-addicts. In: Loimer N et al., editors. Drug addiction and AIDS. Wien: Springer Verlag; 1991. p. 272-85.

27. Cristal S. Health-care barriers and utilization patterns among intravenous drug users with HIV diseases. AIDS Public Policy J 1992; 7:187-98.

28. Des Jarlais DC, Hagan H, Friedman SR et al. Mantaining low HIV seroprevalence in populations of injecting drug users. J Am Med Assoc 1995; 274:1226-31.

29. Telles PR. Perspectivas do programa de redução de danos no Rio de Janeiro. In: Inen C, Baptista M, organizadores. Toxicomanias:abordagem multidisciplinar. Rio de Janeiro: Sette Letras; 1997. p. 149-58. 